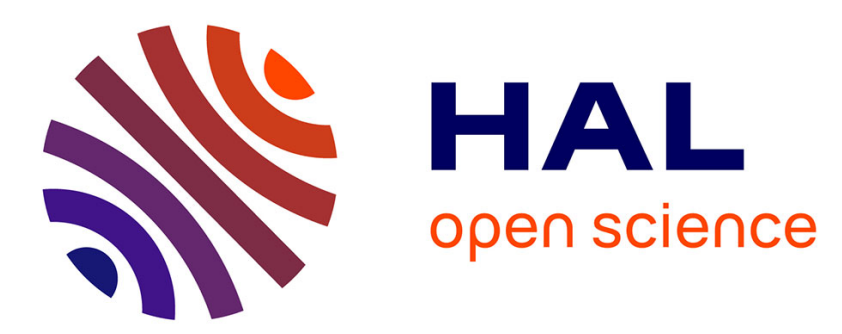

\title{
Analysis of dynamics of liquid jet injected into gaseous crossflow using proper orthogonal decomposition
}

Anirudh Asuri Mukundan, Thibaut Ménard, Marcus Herrmann, Jorge César

Brändle de Motta, Alain Berlemont

\section{- To cite this version:}

Anirudh Asuri Mukundan, Thibaut Ménard, Marcus Herrmann, Jorge César Brändle de Motta, Alain Berlemont. Analysis of dynamics of liquid jet injected into gaseous crossflow using proper orthogonal decomposition. AIAA SciTech 2020 Forum, Jan 2020, Orlando, FL, United States. pp.2020-0357, $10.2514 / 6.2020-0357$. hal-02447945

\section{HAL Id: hal-02447945 \\ https://hal.science/hal-02447945}

Submitted on 21 Jan 2020

HAL is a multi-disciplinary open access archive for the deposit and dissemination of scientific research documents, whether they are published or not. The documents may come from teaching and research institutions in France or abroad, or from public or private research centers.
L'archive ouverte pluridisciplinaire HAL, est destinée au dépôt et à la diffusion de documents scientifiques de niveau recherche, publiés ou non, émanant des établissements d'enseignement et de recherche français ou étrangers, des laboratoires publics ou privés. 


\title{
Analysis of dynamics of liquid jet injected into gaseous crossflow using proper orthogonal decomposition
}

\author{
Anirudh Asuri Mukundan* and Thibaut Ménard ${ }^{\dagger}$ \\ CNRS UMR6614-CORIA, Saint-Étienne-du-Rouvray Cedex 76801, France \\ Marcus Herrmann* \\ SEMTE, Arizona State University, Tempe, AZ 85281, USA \\ Jorge César Brändle de Motta ${ }^{\S}$ and Alain Berlemont ${ }^{\mathrm{II}}$ \\ CNRS UMR6614-CORIA, Saint-Étienne-du-Rouvray Cedex 76801, France
}

In this paper we present the results from the proper orthogonal decomposition (POD) on the liquid volume fraction scalar field from the detailed numerical simulations of turbulent liquid jet injection $(q=6.6, \mathbf{R e}=14000, \mathbf{W e}=2178)$ into subsonic crossflowing air $(\mathbf{R e}=$ 570000, We $=330$ ) to study the waves formed on the windward side of the liquid jet. The liquid/gas interface is captured using a coupled level set volume of fluid (CLSVOF) method. The method of snapshots technique is used in the POD to extract the proper orthogonal modes. A binarized form of the liquid volume fraction is given as input to POD. The modal energies and the least square truncation errors are presented for all the modes considered in this study. A travelling wave pattern is observed in the modes 5 and 6 on the windward side of the liquid jet. The waves in mode 5 are found to be $90^{\circ}$ out-of-phase with those in mode 6 . The approximate wavelength of these waves is in the order of the liquid jet diameter. The orthogonal modes are then used to compute the power spectral density (PSD) and the cross power spectral density (CPSD) to extract spectral information of the system dynamics. The characteristic frequency of mode pairs 5 and 6 is found to be $15.8 \mathrm{kHz}$. The out-of-phase observation is validated by the approximate match of the frequency at which $90^{\circ}$ phase angle difference occurs and the characteristic frequency.

\section{Nomenclature}

$\begin{array}{ll}D_{j} & =\text { Liquid jet diameter } \\ \rho_{j} & =\text { Liquid jet density } \\ U_{j} & =\text { Liquid jet velocity } \\ \mu_{j} & =\text { Liquid jet viscosity } \\ \sigma & =\text { Surface tension coefficient } \\ \rho_{c} & =\text { Crossflow gas density } \\ u_{c} & =\text { Crossflow gas velocity } \\ \mu_{c} & =\text { Crossflow viscosity } \\ q & =\text { Momentum flux ratio } \\ \mathrm{We}_{j} & =\text { Jet Weber number } \\ \operatorname{Re}_{j} & =\text { Jet Reynolds number } \\ \mathrm{We}_{c} & =\text { Crossflow Weber number } \\ \operatorname{Re}_{c} & =\text { Crossflow Reynolds number } \\ r_{\rho} & =\text { Liquid-Gas density ratio } \\ r_{\mu} & =\text { Liquid-Gas viscosity ratio }\end{array}$

*PhD Candidate, CNRS UMR6614-CORIA, Saint-Étienne-du-Rouvray Cedex 76801, France, Student Member

†Associate Professor, Université de Rouen Normandy \& CNRS UMR6614-CORIA, Saint-Étienne-du-Rouvray Cedex 76801, France

$\$$ Associate Professor, School for Engineering of Matter, Transport and Energy, Arizona State University, Tempe, AZ 85281, USA

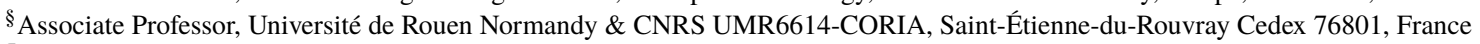
II Research Director, CNRS UMR6614-CORIA, Saint-Étienne-du-Rouvray Cedex 76801, France 
$z_{M, i}=$ Reconstructed image

$\phi_{r} \quad=$ Proper Orthogonal Mode

$a_{r, i}=$ Temporal coefficients

LJIC $=$ Liquid Jet In Crossflow

POD = Proper Orthogonal Decomposition

$\mathrm{POM}=$ Proper Orthogonal Modes

\section{Introduction}

$\mathrm{T}$ HE liquid fuel breakup using aerodynamic forces is the primary driver for atomization in the aero engines. A common technique used in such engines is fuel injection in crossflowing air also called liquid jet in crossflow (LJIC) configuration. Although much of the investigations for such configurations in the past [1-3] have been focused on the investigation of global quantities such as drop size and velocity distribution [4], computation of approximate local drop Weber number [5], the studies on the small scale dynamics are sparse [6]. The jet breakup is observed to occur in the time scale of these small scale dynamics [7]. To that end, proper orthogonal decomposition (POD) has been used in multiple studies, for example Refs. [6, 8], to investigate system dynamics.

POD has been a popular tool for extracting the dynamical structures. Lumley [9] had the seminal contribution towards the application of POD to investigate the coherency of turbulent flow structures. Since then, there has been many works [10-12] that involved extracting, understanding, and analyzing the flow dynamics using POD. A detailed review on the POD can be found in the work of Berkooz et al [13]. Moin and Moser [14] constructed proper orthogonal modes (POM) using data from numerical simulations of a channel flow to compute the characteristic turbulent structures. In fact, POD has been extended to study the dynamics of non-linear fluids and problems involving heat transfer [15] and flow control [16, 17]. Arienti and Soteriou [6] used POD to analyze various jet configurations by using the method of snapshots [18]. In their work, the analysis was performed for understanding the dynamical structures, frequency, and wavelength of these physical structures for LJIC configuration. Various injection conditions have been used to extract the POM in order to study the structures. Herrmann et al. [8] applied the POD using method of snapshots technique on their LJIC simulations to study the dynamics of the liquid jet and the effect of liquid-gas density ratio on the POM. More recently, Leask et al. [11, 12] presented the application of POD and dynamic mode decomposition (DMD) to laminar jet, sinusoidal jet, and LJIC.

In this work, we have performed detailed numerical simulations of LJIC configuration. We have then applied the POD to the liquid volume fraction scalar field to extract the flow structure details. The motivation of this study is to extract and study the dynamical details of the waves formed on the windward side of the liquid jet using POD. Furthermore, as noted, Herrmann et al. [8] has performed one such study using an inlet velocity from in-nozzle large eddy simulations (LES) for liquid phase. In this study, we have imposed a turbulent pipe flow inlet velocity for liquid phase to find the effect and influence of the inlet condition on the proper orthogonal modes (POM). It is to be remarked that a direct comparison between our study and that of Herrmann et al. [8] would not yield significant information due to the vast differences in the inlet conditions in the simulations.

This paper is organized as follows. The governing equations, numerical methods, and schemes used in the flow solver are presented in the section $\Pi$ followed by the presentation of configuration, case setup, and operating conditions in the simulations in section III] Section[IV]present the main steps involved in the POD viz., extraction of orthogonal modes, reconstruction of snapshot from simulations using modes, extraction of spectral information. This is followed by the presentation and discussion of the results in section $\mathrm{V}$ and the concluding remarks.

\section{Governing Equations and Numerical Methods}

An in-house flow solver ARCHER, whose capabilities are described extensively in multiple works [19-|26], is used in this work to solve the following conservative form of the incompressible Navier-Stokes equations

$$
\begin{aligned}
& \boldsymbol{\nabla} \cdot \boldsymbol{u}=0, \\
& \frac{\partial(\rho \boldsymbol{u})}{\partial t}+\boldsymbol{\nabla} \cdot(\rho \boldsymbol{u} \boldsymbol{u})=-\nabla P+\boldsymbol{\nabla} \cdot\left(\mu\left(\nabla \boldsymbol{u}+\nabla^{T} \boldsymbol{u}\right)\right)+\boldsymbol{S}_{\sigma},
\end{aligned}
$$

where $\rho$ is the density, $\boldsymbol{u}$ is the velocity field, $P$ is the pressure field, $\mu$ is the dynamic viscosity, and $\boldsymbol{S}_{\sigma}$ is the force due to surface tension acting on the phase interface. 
The solver ARCHER is structured, parallel and developed for detailed numerical simulations of complex and turbulent multiphase flows with the application to study primary breakup of liquid fuel jets. A staggered variable configuration is used with central finite difference scheme for least numerical dissipation. A projection method as described in Refs. [19, 21] is used for solving the Navier-Stokes equations given above. A $2^{\text {nd }}$ order central difference scheme is employed for discretizing the spatial derivatives to limit dissipation. However, the convection term is discretized using a $5^{\text {th }}$ order WENO scheme to ensure a robust behavior of the solution. A consistent mass and momentum flux computation [20] is employed. The viscous term is discretized following the method described in Sussman et al. [27]. The ghost fluid method (GFM) [28] is employed for the spatial discretization of the Poisson equation for taking into account the force due to surface tension as a pressure jump. The resulting linear system is symmetric and positive definite with five diagonals is solved using a multigrid algorithm for preconditioning a conjugate gradient (CG) method [29]. The temporal derivatives in this study are discretized using a one-step forward Euler scheme.

A coupled level set volume of fluid (CLSVOF) method [19] is used for capturing the liquid/gas interface within the context of multiphase flows. The method uses both level set signed distance function [30] and mass conservative volume of fluid (VOF) [31] to reconstruct the liquid/gas interface. The prominent advantage of this method is the accurate sharp and mass conserving interface reconstruction. A piecewise linear interface calculation (PLIC) method is used in this work for reconstructing the liquid/gas interface. Thus, a linear interface is used as an approximation of the original/reference interface. For more details on the implementation of this numerical method, the reader is referred to Ref. [19].

\section{Case Setup and Configuration}

Detailed numerical simulations of an experimentally validated [32] LJIC configuration is performed in this work. The computational domain size is $40 D_{j} \times 10 D_{j} \times 20 D_{j}$ [5] where $D_{j}=1.3 \mathrm{~mm}$ is the liquid jet diameter. The exit of the liquid jet injector is located at $(0,0,0)$. A sketch of this domain considered in this work is illustrated in Fig. 1 1 along with the boundary conditions.

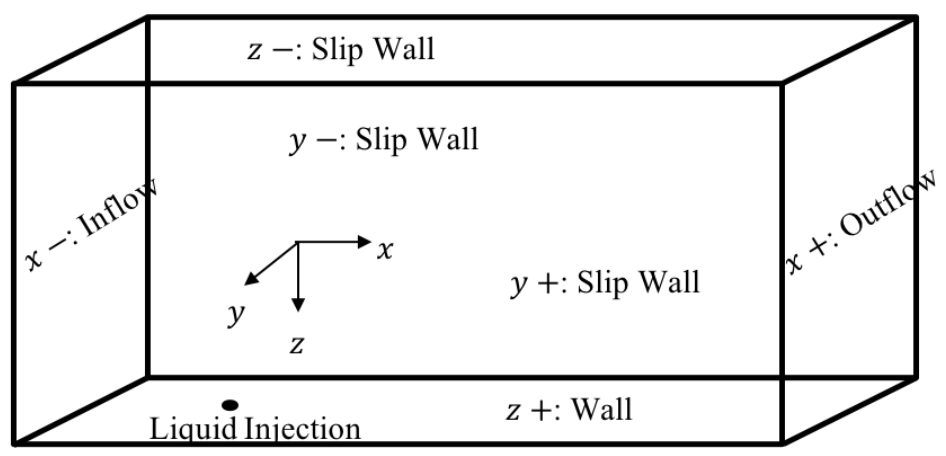

Fig. 1 Illustration of computational domain along with boundary conditions.

The operating conditions used in the simulations are given in Table 1. The non-dimensional numbers such as liquid-gas momentum flux ratio $q$, Weber number (We), and Reynolds number (Re) are kept the same as in the experimental study [32].

A uniform structured Cartesian mesh $1280 \times 320 \times 640$ containing about 262 million cells is used for discretizing the domain resulting in a mesh spacing of $\Delta x=\Delta y=\Delta z=D_{j} / 32$ throughout the domain. We have imposed a fully developed turbulent pipe flow velocity profile to the injected liquid. The simulations are run upto $t^{*}=t U_{j} / D_{j}=73$. It is to be remarked that the employed mesh resolution is not designed to capture the Kolmogorov length scales within the context of turbulent flows. Furthermore, it is to be mentioned that no sub grid scale models are used for the sub grid quantities in the simulations.

\section{Proper Orthogonal Decomposition (POD)}

Figure 2 shows the visualization of the side view of the LJIC configuration with the atomized droplets. It is clearly evident that waves are formed on the windward side of the liquid jet. These waves roll-up, form bag-like structures which subsequently atomize into droplets at the end of the liquid column. Such a breakup is called column breakup. 
Table 1 Operating conditions and non-dimensional numbers

\begin{tabular}{lcc}
\hline \hline Quantity & Unit & Simulation \\
\hline Jet diameter $\left(D_{j}\right)$ & {$[\mathrm{mm}]$} & 1.3 \\
Jet density $\left(\rho_{j}\right)$ & {$\left[\mathrm{kg} / \mathrm{m}^{3}\right]$} & 12.25 \\
Jet velocity $\left(U_{j}\right)$ & {$[\mathrm{m} / \mathrm{s}]$} & 97.84 \\
Jet viscosity $\left(\mu_{j}\right)$ & {$[\mathrm{kg} / \mathrm{ms}]$} & $1.11 \times 10^{-4}$ \\
Surface tension $(\sigma)$ & {$[\mathrm{N} / \mathrm{m}]$} & 0.07 \\
Crossflow gas density $\left(\rho_{c}\right)$ & {$\left[\mathrm{kg} / \mathrm{m}^{3}\right]$} & 1.225 \\
Crossflow gas velocity $\left(u_{c}\right)$ & {$[\mathrm{m} / \mathrm{s}]$} & 120.4 \\
Crossflow viscosity $\left(\mu_{c}\right)$ & {$[\mathrm{kg} / \mathrm{ms}]$} & $1.82 \times 10^{-5}$ \\
& & \\
Liquid-Gas density ratio $\left(r_{\rho}\right)$ & {$[-]$} & 10 \\
Liquid-Gas viscosity ratio $\left(r_{\mu}\right)$ & {$[-]$} & 0.61 \\
Momentum flux ratio $(q)$ & {$[-]$} & 6.6 \\
Jet Weber number $\left(\mathrm{We}_{j}\right)$ & {$[-]$} & 2178 \\
Jet Reynolds number $\left(\mathrm{Re}_{j}\right)$ & {$[-]$} & 14079 \\
Crossflow Weber number $\left(\mathrm{We}_{c}\right)$ & {$[-]$} & 330 \\
Crossflow Reynolds number $\left(\mathrm{Re}_{c}\right)$ & {$[-]$} & 570000 \\
\hline \hline
\end{tabular}

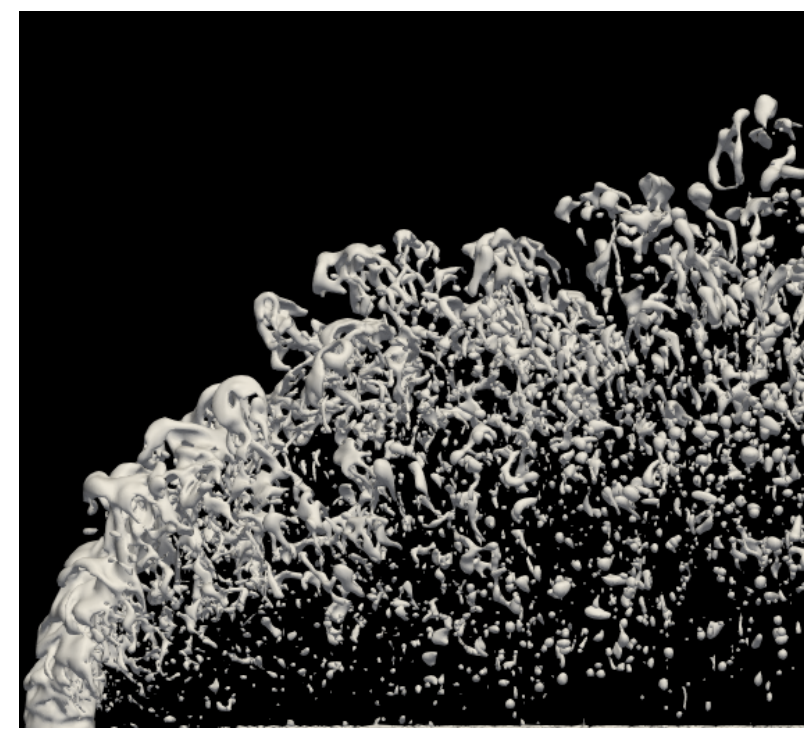

Fig. 2 Visualization of liquid jet from simulations.

These travelling waves have high impact on the atomization characteristics and the system dynamics. We performed simulations with non-turbulent inlet condition for the liquid phase and did not find travelling waves on the windward side of the jet. Thus, it can be remarked that the turbulence in the liquid phase promotes the travelling waves. Furthermore, the interaction between the turbulent liquid and the crossflowing gas phases enhance the formation of these waves on the windward side of the jet. As mentioned in Ref. [6], the time scale of these travelling waves are approximately linked to the time taken by a fluid element to propagate to the top of the liquid column. This time scale is of the order of the aerodynamic characteristic time $t^{\text {aero }}$ which is given as

$$
t^{\text {aero }}=\frac{D_{j}}{U_{j}} \sqrt{\frac{\rho_{j}}{\rho_{c}}} .
$$


This time scale has been observed by Wu et al. [33] to be proportional to the column breakup time scale.

The dynamics of the liquid jet is extracted using the proper orthogonal decomposition (POD) tool. To that end, the method of snapshots technique [18] is utilized. A binarized form of the cumulative liquid volume fraction is given as input to the POD. The liquid volume fraction $F$ is first summed up along the $y$-direction which is then binarized to obtain the input $F^{\text {input }}$ to the POD. The steps in obtaining the $F^{\text {input }}$ is given as follows:

1) $F^{\mathrm{sum}}(x, z)=\int F(x, y, z) d y$,

2) $F^{\text {input }}(x, z)=\left\{\begin{array}{l}1, \text { if } F^{\text {sum }}(x, z)>1 \\ 0, \text { otherwise. }\end{array}\right.$

This binarized liquid volume fraction scalar is obtained for every sampled data written from the simulations to obtain instantaneous snapshots. Each such snapshot is similar to a binarized experimental snapshot wherein it additionally considers volume fraction data on all $y$-planes unlike only the front plane in experiments. The snapshot resolution is $1280 \times 640$ which is same as in simulations. In order to avoid the pollution of the small scale drop structures into POD, the images from the simulations are focused (i.e., cropped) to the near-field region of the liquid jet column. To that end, the resolution of these images is kept to $196 \times 576$ (c.f. Figs. 2 and 3a. The value of $F^{\text {input }}$ will be hereon referred as pixel value, each cell in the $x-z$ plane of the $F^{\text {input }}$ will be referred as pixel, and image from each sampled time step will be referred as snapshot or image.

The method of snapshots technique is then applied to these images from the simulations. In this technique, an optimal set of orthonormal basis functions that span the collection of images/snapshots is obtained. This set of basis is optimal in the sense that it captures the maximum amount of pixel value variation, i.e., energy in the system. These basis functions, denoted as $\phi$, are called proper orthogonal modes (POM). The procedure for the POD implemented in this work follows the study by Arienti and Soteriou [6].

\section{A. Methodology}

An ensemble of $N$ snapshots have been collected from the simulations with $N=38$ in this work. Each image $\left\{x_{i}\right\}, i=1,2, \ldots N$ in this ensemble has resolution of $n \times m$ pixels, where $n=196$ and $m=576$. Each pixel in these snapshots has a resolution of $\Delta x \times \Delta z$ (i.e., snapshots have the same mesh resolution as that of the simulations). These collected image matrices are first converted into vectors [11] and are subsequently put into a matrix $X \in \mathbb{R}^{L \times N}$ where $L=n \times m$, i.e.,

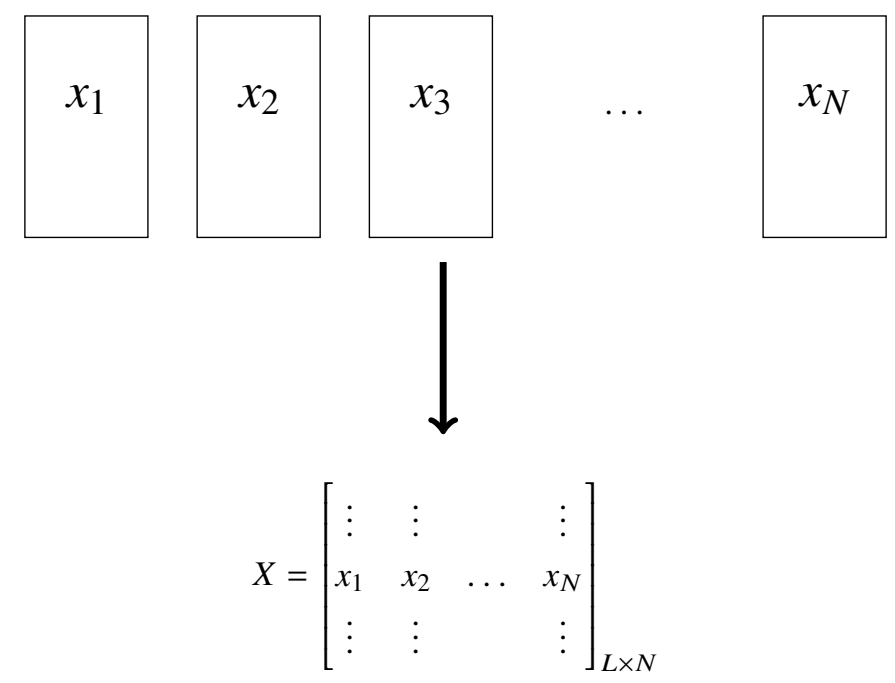

The distance between any two snapshots $x_{f}$ and $x_{g}$ in a sequence of $N$ observations is defined as their inner product $\left\langle x_{f}, x_{g}\right\rangle$, that is, the pixel by pixel sum of the product of the pixel intensities. POD extracts orthonormal basis functions $\phi$ and time dependent orthonormal amplitude coefficients $a_{r, i}$ such that the image reconstruction using the first $M(<N)$ modes is optimal for all the least square truncation errors $\varepsilon_{M}$. These modes are optimal in the sense that, they capture the maximum amount of variation of pixel value with respect to the time average. It is to be remarked that the maximum number of modes that can be used to decompose a system of $N$ observations is $N$, i.e., $M_{\max }=N$. Once the POM and temporal coefficients are obtained, it is possible to reconstruct a snapshot $\left\{x_{i}\right\}$ from simulations at time $t_{i}$ according to 
the following expression,

$$
s_{M, i}=\sum_{r=0}^{M} a_{r, i} \phi_{r}
$$

where $s_{M, i}$ is the image reconstructed using $M$ modes. As a first step, the time average or mean $\phi_{0}$ of all snapshots is computed. It is expressed as

$$
\phi_{0}=\frac{1}{N} \sum_{i=1}^{N} x_{i}
$$

thus, $a_{0, i}=1 \forall i=1,2, \ldots N$. Then the image based pixel value fluctuation is computed as

$$
\widetilde{X}=X-\phi_{0} \equiv \widetilde{x}_{i}=x_{i}-\phi_{0} \quad \forall i=1,2, \ldots N .
$$

A correlation matrix $K\left(\in \mathbb{R}^{N \times N}\right)$ defined as the inner product of the pixel value fluctuation between images. It is computed as

$$
K_{i, j}=\frac{1}{N}\left\langle\widetilde{x_{i}}, \widetilde{x_{j}}\right\rangle
$$

where $\langle\cdot, \cdot\rangle$ represent the inner product. The eigenvalue and eigenvector decomposition of this correlation matrix is performed by solving the linear eigenvalue problem

$$
K v_{r}=\lambda_{r} v_{r}
$$

The POMs $\phi_{r}$ are then obtained from the eigenvectors $v_{r}$ through the relation

$$
\phi_{r}=\frac{1}{\sqrt{N \lambda_{r}}} \sum_{i=1}^{N} v_{r i} \widetilde{x}_{i}, \quad r=1,2, \ldots N .
$$

The eigenvalues $\lambda_{r}$ represent the modal energy of the $r^{\text {th }}$ POM and their contribution towards the total dynamics of the system. This refers to the amount of energy of the system captured by the corresponding mode with respect to the time average $\phi_{0}$. The eigenvectors are orthonormal and the eigenvalues are real and non-negative due to the self-adjoint and non-negative properties of $K[6]$.

The temporal coefficients $a_{r, i}\left(\in \mathbb{R}^{N \times N}\right)$ are obtained by projecting the image sequence of the pixel value on the POMs, i.e.,

$$
a_{r, i}=\left\langle\widetilde{x}_{i}, \phi_{r}\right\rangle, \quad i=1,2, \ldots N .
$$

The least square truncation error $\varepsilon_{M}$ can be expressed in terms of the eigenvalues as

$$
\varepsilon_{M}=\frac{\sum_{r=M+1}^{N} \lambda_{r}}{\sum_{r=1}^{N} \lambda_{r}} .
$$

Hence, by this expression, $\varepsilon_{0}=1$ when only the time averaged snapshot is used and $\varepsilon_{N}=0$ when all $N$ modes are used. The dependence of $\varepsilon_{M}$ on the eigenvalues $\lambda_{r}$ shows that the system dynamics could be reduced to the first few modes (under a good approximation) when there is a significantly large gap between the first few eigenvalues with respect to the rest.

\section{B. Spectral Data Extraction}

In order to analyze the temporal coefficients in the frequency domain, the power spectral density (PSD) for the time series $\left\{a_{i}\right\} \forall i=1,2, \ldots N$ is computed. PSD gives the information whether a mode appears periodically in time or not in a data sequence. It is computed as

$$
\operatorname{PSD}=\frac{1}{N}\left|\sum_{i=0}^{N-1} a_{i} e^{-j 2 \pi f_{s} i / N}\right|^{2},
$$


where $f_{s}$ is the sampling frequency from the simulations. The PSD is computed in the similar fashion as that of Arienti and Soteriou [6] using the Welch's averaged periodogram method [34] with Hanning window of length $N_{H}=18$ having a window overlap of length $N_{H} / 2$.

Furthermore, the temporal correlation of a mode pair $\left\{a_{i}\right\}$ and $\left\{b_{i}\right\}(i=1,2, \ldots N)$ is quantified by the cross power spectral density (CPSD) function. It is computed as

$$
\mathrm{CPSD}=\frac{1}{N} \sum_{i=0}^{N} a_{i} b_{i} e^{-j 2 \pi f_{s} i / N} .
$$

It is to be remarked that the CPSD carries both the amplitude and phase information of the two time series. Within this work, the CPSD is computed using the Welch's averaged periodogram method using Hanning window of $N_{H}=16$.

\section{Results}

The total mass in the simulation computational domain is found to be stabilized at $t^{*}=t U_{j} / D_{j}=28$. Since POD (using the method of snapshots) works best for statistically stationary images, a total of 38 snapshots after $t>28$ are collected. The number of snapshots considered in this work does not represent a converged set of snapshots in statistical point of view. The results shown in this section are obtained using 20 modes unless otherwise specified explicitly.

\section{A. Proper Orthogonal Modes}

The POM are extracted using the procedure described in Section IV.A The zeroth mode $\phi_{0}$ correspond to the time averaged pixel intensities of all the snapshots. Figure 3 shows various instantaneous snapshots from simulations and the zeroth POM.

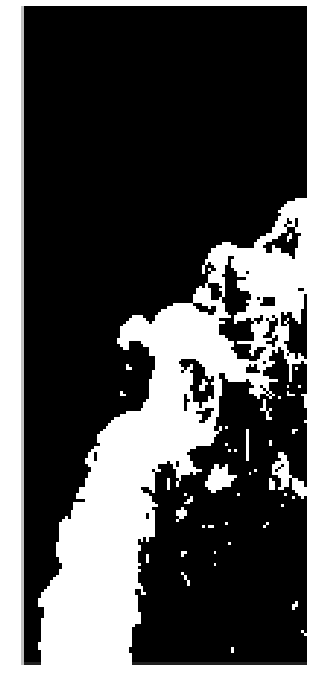

(a) $t^{*}=29.88$

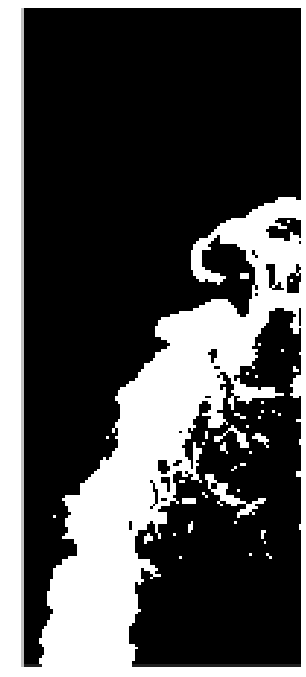

(b) $t^{*}=31.24$

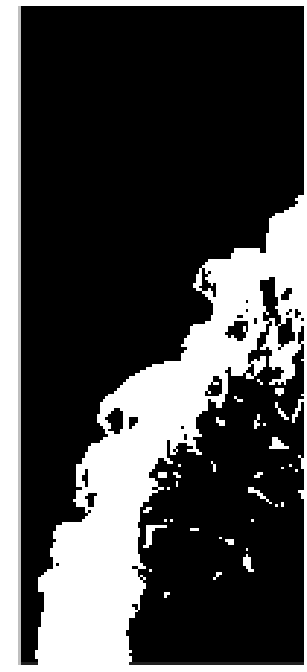

(c) $t^{*}=32.60$

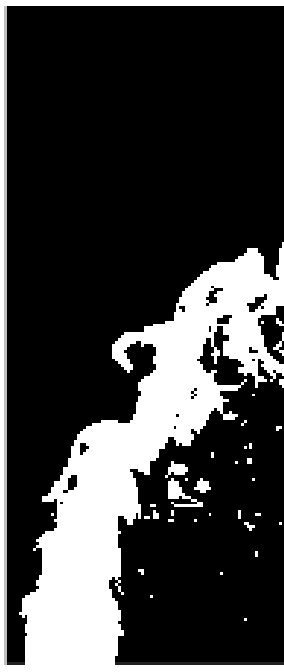

(d) $t^{*}=33.92$

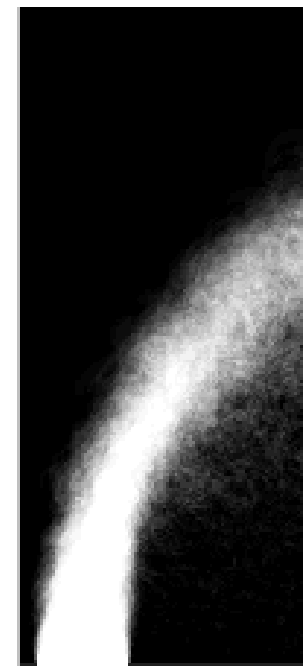

(e) $\phi_{0}$

Fig. 3 Instantaneous snapshots from simulations and zeroth proper orthogonal mode $\left(\phi_{0}\right)$.

Figure 4 shows the first six POM obtained from the POD. The travelling waves on the windward side of the jet discussed in section IV] is recalled here. The red region in the plots of the POM represent the crest of a wave while the blue region indicate the trough of a wave. Thus, a consecutive red and blue region is considered as a wave within the context of this work. The coloring scheme in Fig. 4 is in the RGB scale between 0 and 255. One such travelling wave pattern is visible in the modes 5 and 6 (c.f. Figs. $4 \mathrm{e}$ and $4 \mathrm{f}$ ). It can be observed that these waves are approximately $90^{\circ}$ out-of-phase with each other. It is because the spatial location of the crest (red region) of a wave is in between the crest (red region) and trough (blue region) of a wave in Fig. 5 on the windward side of the jet. This is shown by the dashed lines in Fig.5. Thus, these waves are approximately half-a-wavelength $(\Lambda / 2)$ apart. 


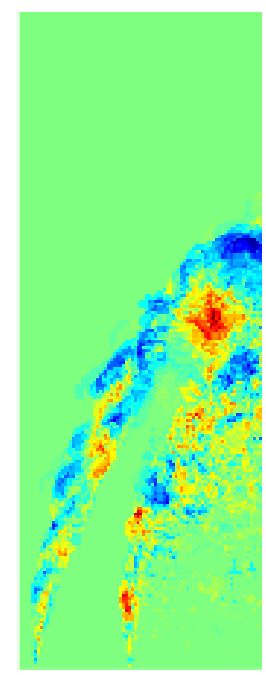

(a) $\phi_{1}$

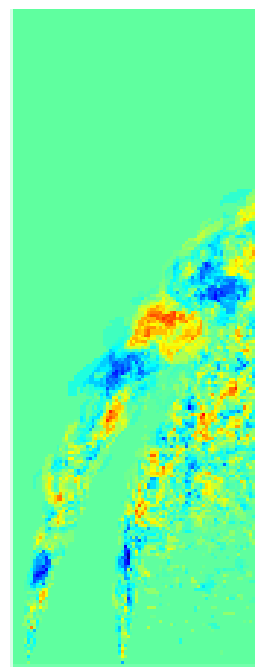

(b) $\phi_{2}$

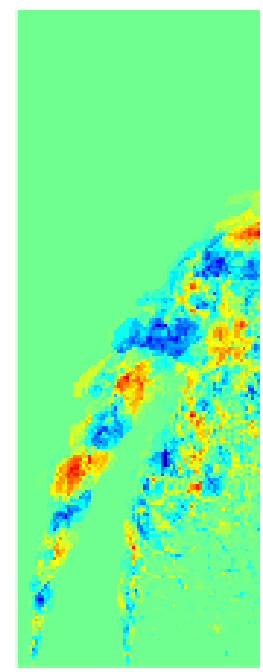

(c) $\phi_{3}$

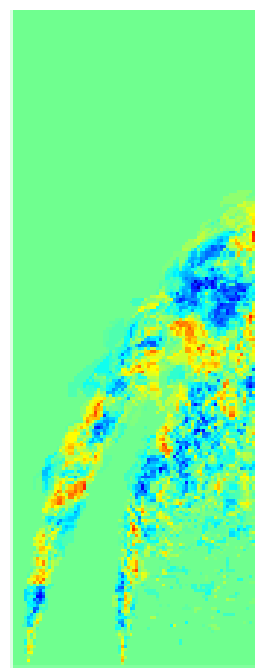

(d) $\phi_{4}$

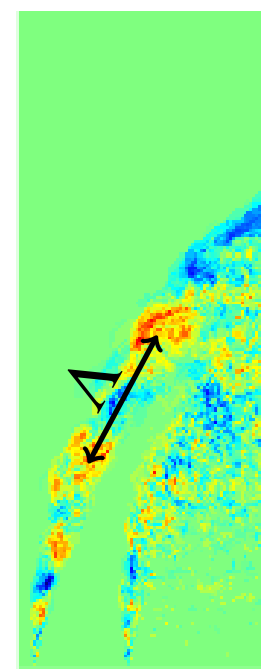

(e) $\phi_{5}$

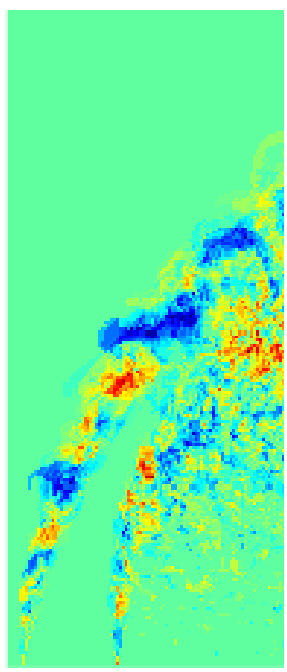

(f) $\phi_{6}$

Fig. 4 First six proper orthogonal modes.

It is to be remarked that when crests (red region) of two travelling waves are located at the same spatial location, they are $0^{\circ}$ out-of-phase whereas they are $180^{\circ}$ out-of-phase when the crest (red region) and trough (blue region) are at the same spatial location.

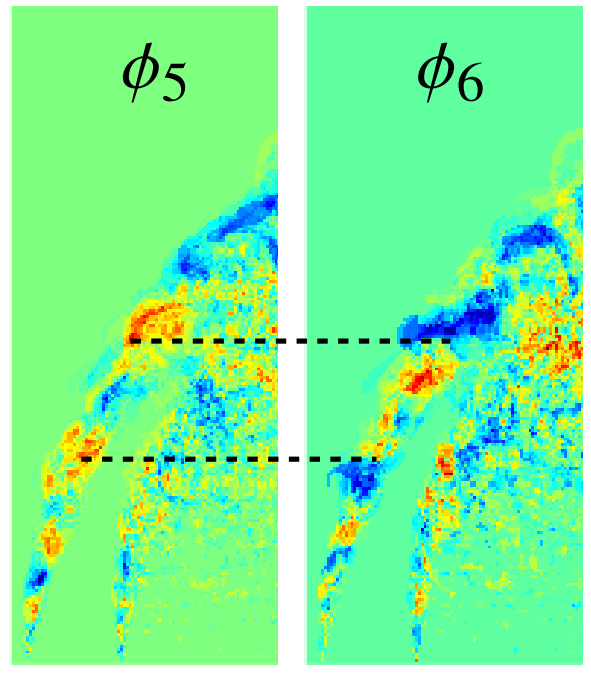

Fig. 5 Phase difference of $90^{\circ}$ between travelling waves on $\phi_{5}$ and $\phi_{6}$.

The wavelength of these waves are measured as an approximate linear Eucledian distance between the center of two consecutive red (or blue) regions shown by solid line in Fig. 4e. The non-dimensionalized approximate wavelength is measured to be $\Lambda_{\phi_{5}-\phi_{6}} / D_{j} \approx 1$. It is remarked that an improved technique to measure the wavelength would yield more accurate values. Herrmann et al. [8] found the wavelength for the same density ratio studied in this work to be $\Lambda / D_{j}=1.7$. This difference in wavelength for the same LJIC configuration could be attributed to the difference in the liquid phase inlet conditions used in their work in comparison to this work.

\section{B. Modal Energy and Least Square Truncation Error}

Each POM carry energy associated with the system dynamics. This is computed using the eigenvalue of the correlation matrix (c.f. Section IV.A). To compute the metric of energy contribution towards the system dynamics, a 
quantity called normalized modal energy is computed using the eigenvalues of the correlation matrix $K$ (c.f. Eq. (10)). It is expressed as

$$
\text { Normalized Modal Energy }=\frac{\lambda_{r}}{\sum_{r=1}^{N} \lambda_{r}} .
$$

In order to find the error in the image reconstruction using $M$ modes, least square truncation error $\varepsilon_{M}$ is computed (c.f. Eq. (12)). Figures $6 \mathrm{a}$ and $6 \mathrm{~b}$ show respectively the plots of the normalized modal energy and least square truncation error for all modes $M=1,2, \ldots 20$. In general, it can be stated that a sharp decrease in the value of $\varepsilon$ after $r$ modes indicates that these modes capture the maximal dynamics of the system. In this case, we do not observe such strong separation both in the normalized modal energy plot (Fig. 6a) least square truncation error plot (Fig. 6b). Such an observation could be attributed to two reasons: first, the continuous nature of the turbulent scales of motion present in the simulations although this claim needs to be validated; second, the lack of convergence of statistical convergence from the simulation snapshots. Therefore, for a more detailed study of the system dynamics of LJIC configuration, convergence of the statistics in the snapshots from simulations is essential and beneficial.

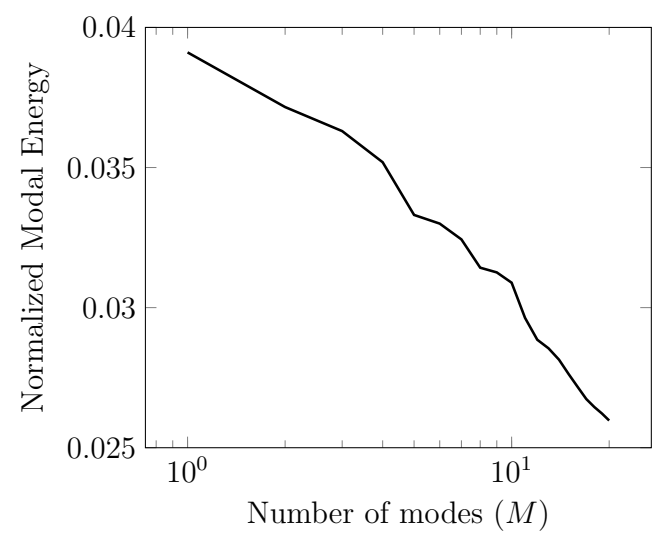

(a) Normalized modal energy.

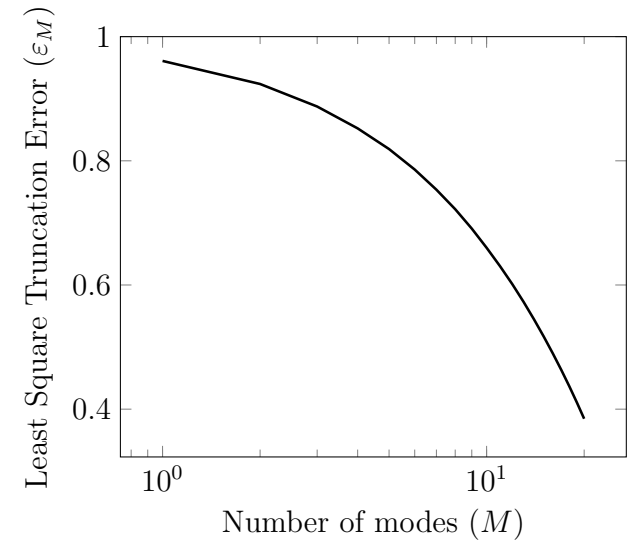

(b) Least square truncation.

Fig. 6 Evolution of normalized modal energy and least square truncation error with mode number.

\section{Image Reconstruction}

One of the advantages of extracting the POM is that any snapshot from the simulations can be reconstructed using few modes that carry majority of the system's energy (c.f. Section IV.A. As shown in Eq. (5), a snapshot $\left\{x_{i}\right\}$ from the simulations can be reconstructed using the POM $\phi_{r}$ and the temporal coefficients $a_{r, i}$. In order to validate it, the snapshot at time $t^{*}=29.88$ (snapshot $\left\{x_{1}\right\}$ ) is considered. To emphasize the importance on the contribution of the total number of modes used for reconstruction, Fig. 7 shows the reconstructed image obtained using 5, 10, 15, and 20 modes. It can be clearly observed that inclusion of more number of modes for image reconstruction captures more details of the jet.

\section{Liquid Jet Spectral Information}

Finally, in order to analyze the dynamics of the liquid jet, the travelling waves on the windward side of the liquid jet are studied. The modes $\phi_{5}$ and $\phi_{6}$ presented in section V.A with travelling wave pattern are considered for spectral information extraction. To that end, the analysis is shifted to the frequency space. The power spectral density (PSD) and cross power spectral density (CPSD) are computed for the first simulation snapshot $\left\{x_{1}\right\}$ for the temporal coefficients $a_{5}$ and $a_{6}$. The sampling frequency $f_{s}$ for the computation of PSD and CPSD (refer Eqs. (13) and (14)) is approximately $55.6 \mathrm{kHz}$ from the simulations. A preliminary analysis reveal that the the PSD spectrum for the $\phi_{5}$ and $\phi_{6}$ peaks at around $13 \mathrm{kHz}$ and $18 \mathrm{kHz}$ as shown by dashdotted and dotted lines in Fig. $8 \mathrm{c}$. The characteristic frequency of this mode pair is defined as the value of frequency at which CPSD amplitude reaches its maximum. However, we observe 


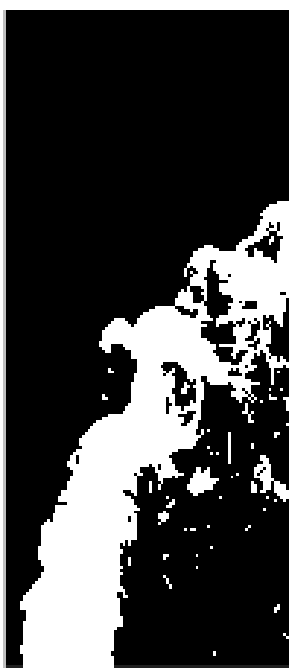

(a) Simulations

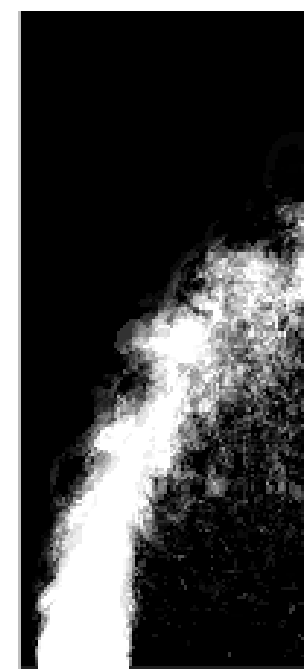

(b) 5 modes

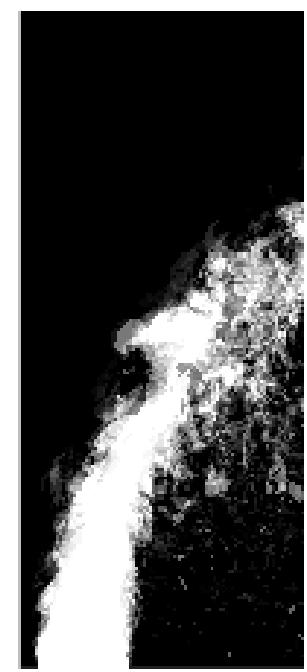

(c) 10 modes

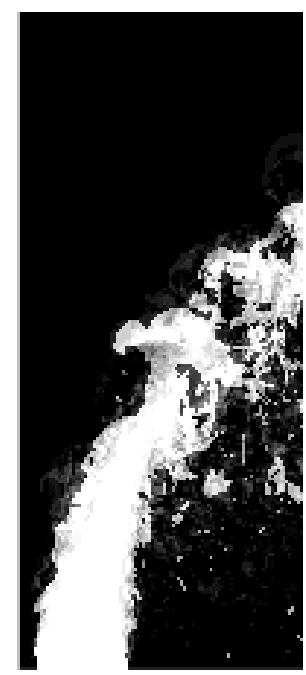

(d) 15 modes

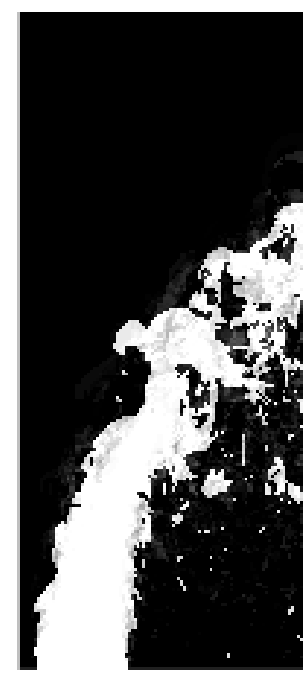

(e) 20 modes

Fig. 7 Image reconstruction using 5, 10, 15, and 20 modes for the snapshot from simulations at $t^{*}=29.88$.

two peaks in Fig. 8d. In this work, the frequency of the global maxima is considered as characteristic frequency which is reported as $15.8 \mathrm{kHz}$ indicated by the dashed line in Fig. $8 \mathrm{~d}$.

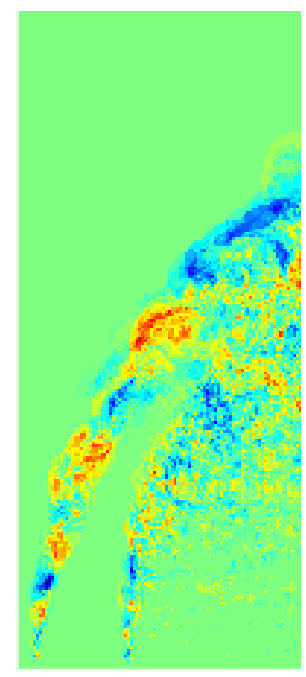

(a) $\phi_{5}$

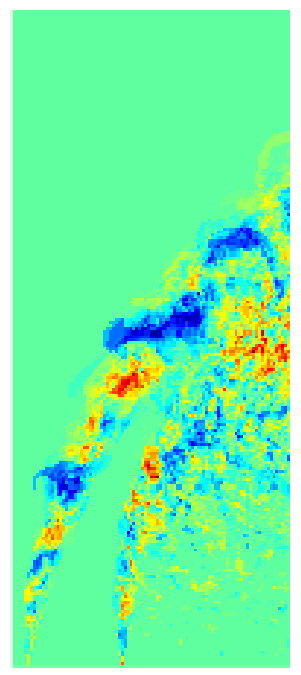

(b) $\phi_{6}$

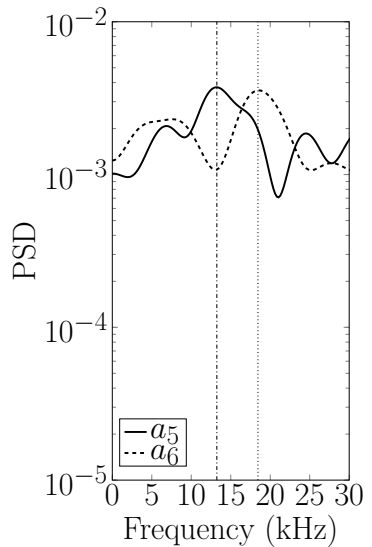

(c) PSD Amplitude

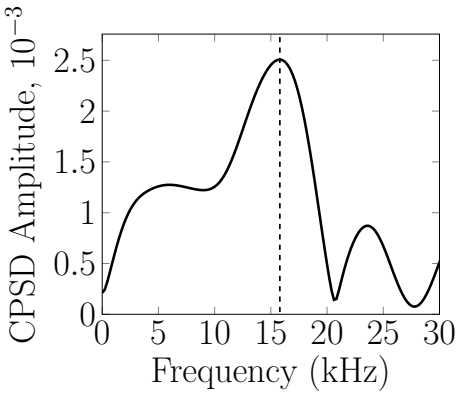

(d) CPSD Amplitude

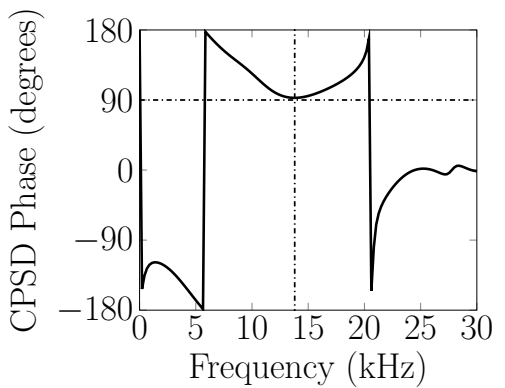

(e) CPSD Phase Angle

Fig. 8 Snapshot of first image $(i=1)$ from simulations, PSD, and CPSD amplitude and phase angle of $a_{5}$ and $a_{6}$ temporal coefficients.

The $90^{\circ}$ phase difference between the travelling waves, as discussed in section V.A (see Fig. 5), is depicted in the Fig. $8 \mathrm{e}$ as the dashed lines. The frequency at which the maximum CPSD amplitude $(15.8 \mathrm{kHz})$ matches approximately the frequency at which the $90^{\circ}$ phase difference occurs $(13.8 \mathrm{kHz})$. 


\section{Conclusions}

Results from proper orthogonal decomposition (POD) of liquid volume fraction data from detailed numerical simulations of liquid jet in crossflow have been presented. The simulations with the turbulent liquid jet injected into subsonic gaseous crossflow has been performed with a liquid-gas density ratio of 10. The liquid/gas interface within the context of multiphase flows has been captured and reconstructed using a coupled level set volume of fluid (CLSVOF) method. A binarized form of the cumulative liquid volume fraction scalar from the simulations has been used as input to the POD which uses method of snapshots technique. The modal energies and the least square truncation error have been presented for various modes along with the simulation snapshot reconstruction. There has been no sharp decrease observed either for the evolution of modal energy nor for least square truncation error with increasing number of modes. This could be attributed to the continuous nature of the turbulent scales of motion present in the simulations and lack of statistical convergence of simulation snapshots. A comparison between the mode 5 and 6 have been made through the observation of a travelling wave pattern on the windward side of the jet. These waves are observed to be $90^{\circ}$ out-of-phase with an approximate wavelength of the same order as the liquid jet diameter. Finally, the spectral information from the POD have been extracted through power spectral density (PSD) and cross power spectral density (CPSD) calculations. A preliminary analysis of the spectral data revealed characteristic frequency of the fifth and sixth mode pair has been observed to be $15.8 \mathrm{kHz}$. Furthermore, the phase angle difference between the travelling waves in mode 5 and 6 is validated to be $90^{\circ}$ since the frequency at which this phase angle difference occurs approximately matches the characteristic frequency.

Future works include the generation and utilization of statistically converged dataset, detailed spectral analysis and validation; and investigating the density and viscosity ratio effect on the proper orthogonal modes (POM).

\section{Acknowledgments}

The funding for this project from the European Union's Horizon 2020 research and innovation programme under the Marie Skłodowska-Curie grant agreement $\mathrm{N}^{\circ} 675676$ is gratefully acknowledged. The computing time at CRIANN (Centre Régional Informatique et d'Applications Numériques de Normandie) under the scientific project No. 2003008 is also gratefully acknowledged. The author Anirudh Asuri Mukundan acknowledges the fruitful interactions with Dr. Marco Arienti, Dr. Fabien Thiesset, Dr. Kuppuraj Rajamanickam and Ms. Louise Cottier during the development of POD subroutines and functions.

\section{References}

[1] Aalburg, C., van Leer, B., Faeth, G. M., and Sallam, K. A., "Properties of nonturbulent round liquid jets in uniform gaseous cross flows," Atomization and Sprays, Vol. 15, No. 3, 2005, pp. 271-294. doi:10.1615/AtomizSpr.v15.i3.20.

[2] Ng, C.-L., Sankarakrishnan, R., and Sallam, K. A., "Bag breakup of nonturbulent liquid jets in crossflow," International Journal of Multiphase Flow, Vol. 34, No. 8, 2008, pp. 241-259. doi:10.1016/j.ijmultiphaseflow.2007.07.005, URL https: //doi.org/10.1016/j.ijmultiphaseflow.2007.07.005

[3] Broumand, M., Ahmed, M. M. A., and Birouk, M., "Experimental investigation of spray characteristics of a liquid jet in a turbulent subsonic gaseous crossflow," Proceedings of the Combustion Institute, Vol. 37, No. 3, 2019, pp. 3237-3244.

[4] Herrmann, M., "The influence of density ratio on the primary atomization of a turbulent liquid jet in crossflow," Proceedings of the Combustion Institute, Vol. 33, No. 2, 2011, pp. 2079-2088. doi:10.1016/j.proci.2010.07.002, URL https://doi .org/10. $1016 /$ j.proci.2010.07.002

[5] Asuri Mukundan, A., Ménard, T., Berlemont, A., Brändle de Motta, J. C., and Herrmann, M., "Validation and Analysis of primary atomization of turbulent liquid jet in crossflow simulations," Proceedings of the ILASS Americas, 30th Annual Conference on Liquid Atomization and Spray Systems, May 12-15, Phoenix, AZ, USA, 2019, 2019.

[6] Arienti, M., and Soteriou, M. C., "Time-resolved proper orthogonal decomposition of liquid jet dynamics," Physics of Fluids, Vol. 21, No. 112104, 2009. doi:10.1063/1.3263165, URL https://doi-org.ezproxy1.lib.asu.edu/10.1063/ 1.3263165

[7] Less, D. M., and Schertz, J. A., "Transient behavior of liquid jets injected normal to a high-velocity gas stream," AIAA Journal, Vol. 24, No. 12, 1986, pp. 1979-1986. doi:10.2514/3.9556, URL https://doi.org/10.2514/3.9556

[8] Herrmann, M., Arienti, M., and Soteriou, M. C., “The Impact of Density Ratio on the Liquid Core Dynamics of a Turbulent Liquid Jet Injected Into a Crossflow,” Journal of Engineering for Gas Turbines and Power, 2011. doi:10.1115/1.4002273. 
[9] Lumley, J. L., Atmospheric Turbulence and Radio Wave Propagation, Nauka, Moscow, 1967, pp. 166-178.

[10] Schmid, P. J., "Dynamic mode decomposition of numerical and experimental data," Journal of Fluid Mechanics, Vol. 656, 2010, pp. 5-28. doi:10.1017/S0022112010001217.

[11] Leask, S. B., McDonell, V. G., and Samuelsen, S., "Understanding the Physical Interpretation of Proper Orthogonal Decomposition and Dynamic Mode Decomposition for Liquid Injection," 11th U.S. National Combustion Meeting, April 24th-27th, Pasadena, California, USA, 2019. URL https://wssci.us/meetings/ncm2019/papers/3G06.pdf

[12] Leask, S. B., McDonell, V. G., and Samuelsen, S., "An Analysis of Proper Orthogonal Decomposition and Dynamic Mode Decomposition on Liquid Jets in Crossflow," Proceedings of the ILASS Americas, 30th Annual Conference on Liquid Atomization and Spray Systems, May 12-15, Phoenix, AZ, USA, 2019, 2019. URL http://www.ilass.org/2/conferencepapers/87_ 2019.pdf

[13] Berkooz, G., Holmes, P., and Lumley, J. L., "The proper orthogonal decomposition in the analysis of turbulent flows," Annual Review of Fluid Mechanics, Vol. 25, 1993, pp. 539-575. doi:10.1146/annurev.fl.25.010193.002543, URL https: //doi.org/10.1146/annurev.fl.25.010193.002543.

[14] Moin, P., and Moser, R. D., "Characteristic eddy decomposition of turbulence in a channel," Journal of Fluid Mechanics, Vol. 200, 1989, pp. 471-509. doi:10.1017/S0022112089000741, URL https://doi.org/10.1017/S0022112089000741

[15] Park, H. M., and Cho, D. H., "The use of the Karhunen-Loeve decomposition for the modeling of distributed parameter systems," Chemical Engineering Science, Vol. 51, No. 1, 1996, pp. 81-98. doi:10.1016/0009-2509(95)00230-8, URL https://doi.org/10.1016/0009-2509(95)00230-8

[16] Park, H. M., and Lee, M. W., "An efficient method of solving the Navier-Stokes equation for flow control," International Journal of Numerical Methods in Engineering, Vol. 41, No. 6, 1998, pp. 1133-1151. URL https://doi .org/10.1002/(SICI) 1097$0207(19980330) 41: 6<1133:$ :AID-NME329>3 . 0 . C0;2-Y

[17] Rediniotis, O. K., Ko, J., Yue, X., and Kurdila, A. J., "Synthetic jets, their reduced order modeling and applications to flow control," 37th AIAA Aerospace Sciences Meeting and Exhibit, Reno, NV, 1999.

[18] Sirovich, L., "Turbulence and the Dynamics of Coherent Structures," Quarterly of Applied Mathematics, Vol. 45, No. 3, 1987, pp. 561-571.

[19] Ménard, T., Tanguy, S., and Berlemont, A., "Coupling level set/VOF/ghost fluid methods: Validation and application to 3D simulation of the primary break-up of a liquid jet," International Journal of Multiphase Flow, Vol. 33, No. 5, 2007 , pp. 510-524. doi:10.1016/j.ijmultiphaseflow.2006.11.001, URL https://doi .org/10.1016/j . ijmultiphaseflow.2006.11.001

[20] Vaudor, G., Ménard, T., Aniszewski, W., Doring, M., and Berlemont, A., "A consistent mass and momentum flux computation method for two phase flows. Application to atomization process," Computers \& Fluids, Vol. 152, 2017, pp. $204-216$. doi:10.1016/j.compfluid.2017.04.023, URL https://doi.org/10.1016/j.compfluid.2017.04.023

[21] Asuri Mukundan, A., Ménard, T., Brändle de Motta, J. C., and Berlemont, A., "A 3D Moment of Fluid method for simulating complex turbulent multiphase flows," Computers \& Fluids, Vol. 198, 2020. doi:10.1016/j.compfluid.2019.104364, URL https://doi.org/10.1016/j.compfluid.2019.104364

[22] Asuri Mukundan, A., Ménard, T., Berlemont, A., and Brändle de Motta, J. C., "Coupled Level set moment of fluid method for simulating multiphase flows," Proceedings of the ILASS Europe, 29th Annual Conference on Liquid Atomization and Spray Systems, September 2-4, Paris, France, 2019, 2019.

[23] Asuri Mukundan, A., Ménard, T., Berlemont, A., and Brändle de Motta, J. C., "An investigation of characteristics of airblast atomization using 3D DNS for altitude relight conditions," 11th U.S. National Combustion Meeting, April 24th-27th, Pasadena, California, USA, 2019. URL https://wssci.us/meetings/ncm2019/papers/

[24] Asuri Mukundan, A., Ménard, T., Berlemont, A., and Brändle de Motta, J. C., "A comparative study of DNS of airblast atomization using CLSMOF and CLSVOF methods," Proceedings of the ILASS Europe, 29th Annual Conference on Liquid Atomization and Spray Systems, September 2-4, Paris, France, 2019, 2019.

[25] Asuri Mukundan, A., Ménard, T., Berlemont, A., Brändle de Motta, J. C., and Eggels, R., "Validation and Analysis of 3D DNS of planar pre-filming airblast atomization simulations," Proceedings of the ILASS Americas, 30th Annual Conference on Liquid Atomization and Spray Systems, May 12-15, Phoenix, AZ, USA, 2019, 2019. 
[26] Chéron, V., Brändle de Motta, J. C., Vaudor, G., Ménard, T., and Berlemont, A., "From droplets to particles: Transformation criteria," Proceedings of the ILASS Europe, 29th Annual Conference on Liquid Atomization and Spray Systems, September 2-4, Paris, France, 2019, 2019.

[27] Sussman, M., Smith, K. M., Hussaini, M. Y., Ohta, M., and Zhi-Wei, R., "A sharp interface method for incompressible two-phase flows," Journal of Computational Physics, Vol. 221, No. 2, 2007, pp. 469-505. doi:10.1016/j.jcp.2006.06.020, URL https://doi.org/10.1016/j.jcp.2006.06.020

[28] Fedkiw, R., Aslam, T., Merriman, B., and Osher, S., “A Non-oscillatory Eulerian Approach to Interfaces in Multimaterial Flows (the Ghost Fluid Method)," Journal of Computational Physics, Vol. 152, No. 2, 1999, pp. 457-492. doi:10.1006/jcph.1999.6236, URL https://doi.org/10.1006/jcph.1999.6236

[29] Tanguy, S., and Berlemont, A., "Application of a level set method for simulation of droplet collisions," International Journal of Multiphase Flow, Vol. 31, 2005, pp. 1015-1035. doi:10.1016/j.ijmultiphaseflow.2005.05.010, URL https: $/ / 10.1016 /$ j.ijmultiphaseflow.2005.05.010

[30] Osher, S., and Sethian, J. A., "Fronts propagating with curvature-dependent speed: Algorithms based on Hamilton-Jacobi formulations," Journal of Computational Physics, Vol. 79, No. 1, 1988, pp. 12-49. doi:10.1016/0021-9991(88)90002-2, URL https://doi.org/10.1016/0021-9991(88)90002-2

[31] Aulisa, E., Manservisi, S., Scardovelli, R., and Zaleski, S., “A geometrical area-preserving Volume-of-Fluid advection method," Journal of Computational Physics, Vol. 192, No. 1, 2003, pp. 355-364. doi:10.1016/j.jcp.2003.07.003, URL https://doi.org/10.1016/j.jcp.2003.07.003

[32] Brown, C. T., and McDonell, V. G., "Near Field Behavior of a Liquid Jet in a Crossflow," Proceedings of ILASS Americas, 19th Annual Conference on Liquid Atomization and Spray Systems, May 23-26, Toronto, Canada, 2006, 2006.

[33] Wu, P. K., Kirkendall, K. A., Fuler, R. O., and Nejad, A. S., "Breakup Processes of Liquid Jets in Subsonic Crossflows," Journal of Propulsion and Power, Vol. 13, No. 1, 1997, pp. 64-73. doi:10.2514/2.5151, URL https://doi.org/10.2514/2.5151.

[34] Welch, P., "The use of fast Fourier transform for the estimation of power spectra: A method based on time averaging over short, modified periodograms," IEEE Transactions on Audio and Electroacoustics, Vol. 15, No. 2, 1967, pp. 70-73. 